\title{
Dynamic compensation of an rf cavity failure in a superconducting linac
}

\author{
Jean-Luc Biarrotte ${ }^{1, *}$ and Didier Uriot ${ }^{2, \dagger}$ \\ ${ }^{1}$ CNRS, IPN Orsay, 15 rue G. Clémenceau, 91406 Orsay cedex, France \\ ${ }^{2}$ CEA IRFU, Service des Accélérateurs, de Cryogénie et de Magnétisme F-91191 Gif-sur-Yvette, France
}

(Received 5 December 2007; published 24 July 2008)

\begin{abstract}
An accelerator driven system (ADS) for transmutation of nuclear waste typically requires a $600 \mathrm{MeV}$ $1 \mathrm{GeV}$ accelerator delivering a proton flux of a few $\mathrm{mA}$ for demonstrators, and of a few tens of $\mathrm{mA}$ for large industrial systems. Such a machine belongs to the category of the high-power proton accelerators, with an additional requirement for exceptional "reliability": because of the induced thermal stress to the subcritical core, the number of unwanted "beam trips" should not exceed a few per year, a specification that is several orders of magnitude above usual performance. In order to meet this extremely high reliability, the accelerator needs to implement, to the maximum possible extent, a fault-tolerance strategy that would allow beam operation in the presence of most of the envisaged faults that could occur in its beam line components, and in particular rf systems' failures. This document describes the results of the simulations performed for the analysis of the fault-tolerance capability of the XT-ADS superconducting linac in the case of an rf cavity failure. A new simulation tool, mixing transient $\mathrm{rf}$ behavior of the accelerating cavities with full 6D description of the beam dynamics, has been developed for this purpose. Fast fault-recovery scenarios are proposed, and required research and development is identified.
\end{abstract}

PACS numbers: 29.27.Bd, 29.27.Eg, 29.27.Fh, 41.75.-i

\section{INTRODUCTION}

The European Transmutation Demonstration requires a high-power proton accelerator operating in $\mathrm{CW}$ mode, ranging from 1.5 MW (XT-ADS operation, at $600 \mathrm{MeV}$ ) up to $16 \mathrm{MW}$ [European Facility for Industrial Transmutation (EFIT), at $800 \mathrm{MeV}$ ]. A reference design for this accelerator is being developed since 2001 through the preliminary design studies of an experimental accelerator driven system (PDS-XADS) and the EUROTRANS programs, e.g. [1-4]; it is mainly based on the use of a superconducting linac, from at least $20 \mathrm{MeV}$. Such a choice allows one to obtain a very modular and upgradable machine (same concept for prototype and industrial scale), an excellent potential for reliability, and a high rf-to-beam efficiency thanks to superconductivity (optimized operation cost).

The ADS accelerator is also expected-especially in the long term EFIT scenario - to have a very limited number of unexpected beam interruptions per year, which would cause the absence of the beam on the spallation target for times longer than $1 \mathrm{~s}$. This requirement is motivated by the fact that frequently repeated beam interruptions induce thermal stresses and fatigue on the reactor structures, the target, or the fuel elements, with possible significant damages, especially on the fuel claddings; moreover, these beam interruptions decrease the plant availability, implying plant shut-downs in most of the cases. Therefore, it has been estimated that beam trips in excess of $1 \mathrm{~s}$ duration

\footnotetext{
*biarrott@ipno.in2p3.fr

†duriot@cea.fr
}

should not occur more frequently than 5 times per 3-month operation period for the XT-ADS, and 3 times per year for the EFIT.

To reach such an ambitious goal, which is lower than the reliability experience of typical accelerator based user facilities by 2 or 3 orders of magnitude, it is clear that reliability-oriented design practices need to be followed from the early stage of components design. In particular, (i) "strong design" practices are needed: every linac main component has to be derated with respect to its technological limitation (overdesign); (ii) a rather high degree of redundancy needs to be planned in critical areas; this is especially true for the identified "poor-reliability" components: linac injector, and rf power systems, where solidstate amplifiers should be used as much as possible; (iii) fault-tolerance capabilities have to be introduced to the maximum extent: such a capability is expected in the highly modular superconducting rf linac, where any rf cavity or magnet failure could theoretically be compensated using the neighboring elements [5].

Preliminary "integrated reliability" analysis [6], performed in the frame of the PDS-XADS project, show that, using such principles, the number of expected beam trips can be drastically reduced, possibly down to the ADS goal. One of the major contributions to this reliability improvement comes from the implementation of the fault-tolerance principle in a superconducting linac composed with modular rf accelerating cavity systems. Therefore, we propose in this document to investigate in more details the way rf cavity failures can be dynamically compensated in such a superconducting linac. 
TABLE I. General layout of a 5-600 MeV XT-ADS linac using only independently phased SC cavities.

\begin{tabular}{|c|c|c|c|c|c|}
\hline Section number & 1 & 2 & 3 & 4 & 5 \\
\hline Input energy [MeV] & 5 & 16.7 & 90.5 & 191.7 & 498.1 \\
\hline Output energy [MeV] & 16.7 & 90.5 & 191.7 & 498.1 & 614.7 \\
\hline Cavity technology & \multicolumn{2}{|c|}{ Spoke $352.2 \mathrm{MHz}$} & & \multicolumn{2}{|l|}{ Elliptical 704.4 MHz } \\
\hline Structure $\beta$ & 0.15 & 0.35 & 0.47 & 0.65 & 0.85 \\
\hline Number of cavity cells & 2 & 2 & 5 & 5 & 6 \\
\hline Number of cavities & 36 & 60 & 28 & 51 & 12 \\
\hline Focusing type & \multicolumn{5}{|c|}{$\mathrm{NC}$ quadrupole doublet } \\
\hline Cavities/lattice & 2 & 3 & 2 & 3 & 4 \\
\hline Synch phase [deg] & -40 to -27 & -27 to -20 & & -25 & \\
\hline Lattice length [m] & 1.58 & 2.42 & 4.16 & 5.73 & 8.37 \\
\hline Section length [m] & 28.5 & 49.0 & 58.5 & 98.5 & 26 \\
\hline$<$ gradient $>[\mathrm{MeV} / \mathrm{m}]$ & 0.4 & 1.5 & 1.7 & 3.1 & 4.5 \\
\hline
\end{tabular}

\section{SUPERCONDUCTING LINAC AND FAULT TOLERANCE}

\section{A. The XT-ADS reference superconducting linac}

In the present XT-ADS linac reference scheme, a fully modular superconducting linac, based on the use of independently phased rf structures (spoke, elliptical), accelerates the beam from " $X \mathrm{MeV}$ " up to the final energy $(600 \mathrm{MeV})$. This $X \mathrm{MeV}$ energy, which corresponds to the transition between the redundant injector and the fault-tolerant superconducting linac, is still to be optimized between 5 and $50 \mathrm{MeV}$. It will be definitely frozen, probably around $17 \mathrm{MeV}$, at the end of the EUROTRANS program, together with a new optimized linac design for the XT-ADS reference accelerator.

Table I presents, from the PDS-XADS study, a possible layout for the modular superconducting linac, starting from $X=5 \mathrm{MeV}$. This particular accelerator scheme, which corresponds to the largest energy range possible for the independently phased section, will be used to perform the fault-tolerant analysis. More details on this design can be found in [1].

\section{B. The fault-tolerance strategy}

During the PDS-XADS project, systematic beam dynamics simulations in the stationary regime have already been performed on this reference accelerator to analyze the consequences of an rf cavity (or rf power system) failure [1,5]. From this first analysis, it appears that if nothing is done, a cavity's failure leads in nearly all the cases to a complete beam loss, due to the nonrelativistic varying velocity of the particles. To avoid such a total beam loss, it is clear that some kind of retuning has to be performed to compensate the lack of acceleration due to the faulty cavity.

To achieve this compensation, the general philosophy is to readjust the accelerating fields and phases of the nonfaulty accelerating cavities to recover the nominal beam characteristics at the end of the linac, and in particular its energy, while ensuring the same level of transmission as in the reference linac case. This is a "global feedback" concept (see Fig. 1). This can be done using all the cavities downstream of the faulty cavity ("general compensation method"), or only using the accelerating cavities neighboring the failing one ("local compensation method"). In both cases, the retuning procedure, to be fast enough, requires some fast longitudinal beam dynamics simulations and/or the use of a predefined set-points database before being performed. Indeed, relying on longitudinal beam diagnostics (phase detectors, time of flight), as depicted in the ideal feedback scheme of Fig. 1, appears to be far too time consuming to apply in practice.

In the general compensation method, all the linac components downstream of the faulty cavity are retuned, so that the overpower required to compensate the faulty element is shared between a large number of rf cavities: the clear advantage is that this method only requires very small

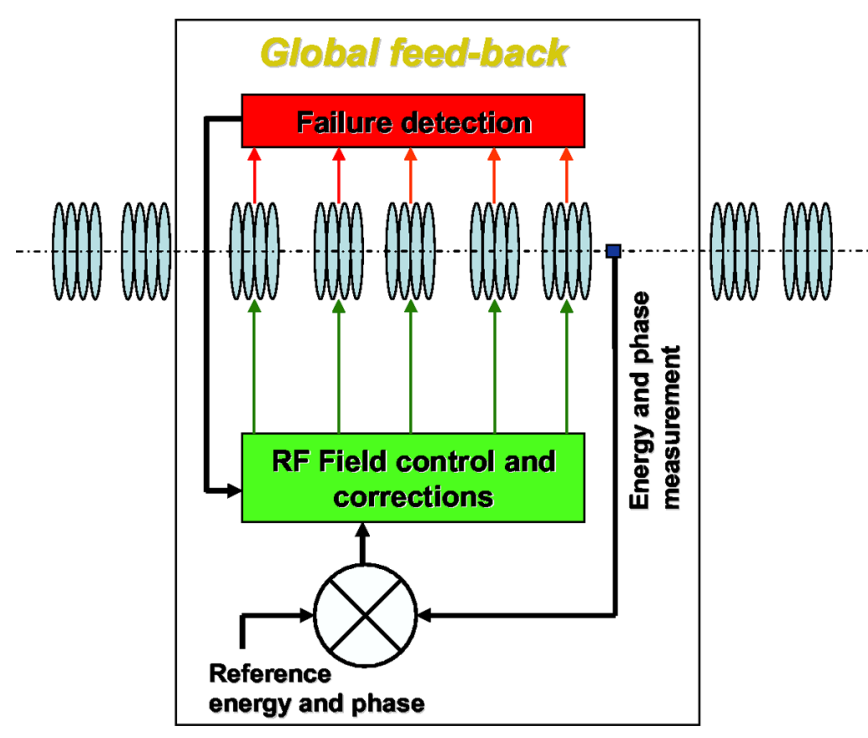

FIG. 1. (Color) Global feedback scheme for rf-fault recovery procedures. 
margins in terms of rf power and accelerating field. But this statement is only valid if the retuning is performed one single time; in order to be able to recover several faulted cavities, the rf margins have to be increased proportionally. On the other hand, the global retuning procedures become more complex than the local one, since involving a high number of elements, and the probability for retuning errors might be higher. On the practical point of view, a very important step has already been reached since this method has been demonstrated on-line at the SNS, where such a fault-recovery system has been recently tested on real operation with low average current high-velocity proton beams $(>200 \mathrm{MeV})$ [7]. The time required to achieve such a retuning is about a few minutes presently, but could probably be lowered to a few seconds or even less than a second [8]. Of course, during the retuning procedure, the beam has to be stopped.

The local compensation method, on the contrary, has the advantage of involving a small number of elements, simplifying the retuning procedures and limiting the possible induced errors. It is illustrated on Fig. 2: if cavity \#n is faulty, the 4 surrounding cavities ( $\#-2, \# n-1, \# n+1$, $\# n+2)$ are retuned to recover the nominal beam energy and phase at the end of the following lattice (point $\mathrm{M}$ ), and by consequence, at the linac end. It can of course be done with more (or less) cavities if necessary. Beam dynamics simulations show that the nominal beam parameters at the target can always be restored using such a retuning method, even if the cavity failure happens at very low energy, given the condition that a $+30 \%$ rise in rf power and accelerating field can be sustained in the cavities [5]. It has to be noted nevertheless that the situation is substantially more difficult to manage in the low-energy section of the linac (below $20 \mathrm{MeV}$ and even more below $10 \mathrm{MeV}$ ), due to the very low velocity of the beam, and consequently to its still quite high nonnormalized (geometrical) longitudinal emittance: in this low-energy case, one has also to take care in the retuning procedure not only of the beam phase and energy, but also of its longitudinal phase advance. The local compensation method is of course demanding in terms of rf power budget, but it is in line with the ADS overdesign criterion, so that it should not lead to dramatic overcosts.

From the previous statements, it appears that some kind of compromise should be found concerning the number of cavities to be used in the recovery procedures. This "optimal" number will have to be searched for, relying especially on a detailed global reliability analysis of the XTADS machine, planned to be performed in the following years, and on practical experimental results later on. But in any case, the retuning philosophy remains the same.

\section{Investigating fast failure recovery scenarios}

The remaining step in the fault-tolerance analysis is now to identify and develop fast failure recovery scenarios to ensure that such retunings can be performed in less than $1 \mathrm{~s}$. Two ways are investigated, but in each case, the very first hypothesis is that, whatever the failure type ( $\mathrm{rf}$ system breakdown, cavity quench ...), the failure can be anticipated early enough (via adequate diagnostics to be defined) to trigger always the same reference recovery scenario, in which the rf power system is forced to shut down to zero while the cavity is still in its superconducting state ("nominal" shutdown).

The first scenario consists in stopping the beam to achieve the retuning (scenario $n^{\circ} 1$ ). - This solution is compatible with both general and local compensation methods, and is already used at the SNS (but with "minutes" beam stops up to now). On the beam dynamics point of view, it has already been demonstrated in the previous PDS-XADS studies dedicated to the stationary fault-tolerance analysis, as mentioned before. This method would probably require practically (i) fast fault anticipation/detection; (ii) beam shutdown, and faulted cavity rf system nominal shutdown; (iii) fast access to a predefined set-point general database, or to the result of an appropriated longitudinal beam dynamics fast simulation; (iv) fast update and tracking of the new low-level rf (LLRF) field and phase set-points; (v) adequate management of the tuner of the failed cavity to put it off frequency and avoid any deceleration effect due to beam loading; (vi) beam reinjection.

All these stages have to last less than $1 \mathrm{~s}$.

The other way is to try to perform the retuning without stopping the beam (scenario $n^{\circ} 2$ ). - This solution, which is a priori more compatible with the local compensation method (minimization of the number of transient adjustments), has to be demonstrated on the beam dynamics point of view to check if it can cope with the probable induced transient beam losses. This transient study is ac-

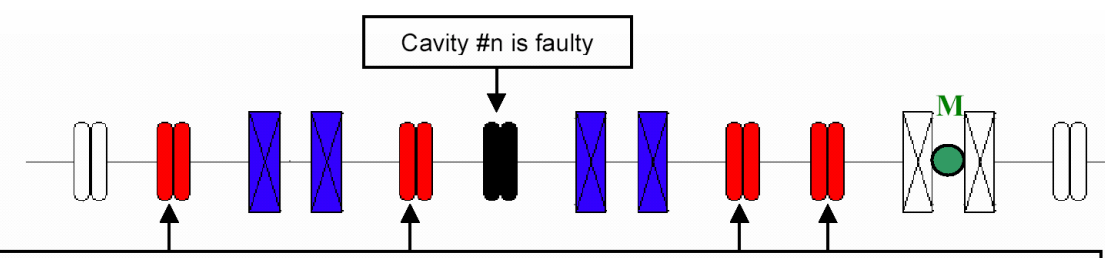

Cavities \#n-2, \#n-1, \#n+1, \#n+2 are retuned to recover the nominal beam energy \& phase at point $M$

FIG. 2. (Color) Principle of the local compensation method. 
tually the main topic of this note. In this case, the procedure would probably require practically (i) very fast fault anticipation/detection; (ii) faulted cavity rf system nominal shutdown; (iii) fast access to a predefined set-points general database; (iv) fast update and tracking of the new field and phase set-points, based on the foreseen failed cavity transient behavior (precalculated tables), to recover quickly the nominal beam transmission and energy; (v) slow update and tracking of the new field and phase set-points with the same method while detuning the failed cavity to avoid the beam loading effect;

The three first stages have to be as fast as possible to avoid significant beam losses.

Finally, one has to note that in every scenario, digital techniques become necessary to meet the speed and software configuration required by such retuning procedures. Field-programmable-gate-array-based digital LLRF control systems, in which a number of key functionalities are implemented on a single chip, can offer such a high-grade reliability and flexibility. Developments are going on in CEA and CNRS at $704 \mathrm{MHz}$ and $352 \mathrm{MHz}$, respectively, in the frame of the EUROTRANS project, with very encouraging preliminary results $[9,10]$.

\section{TRANSIENT BEHAVIORS OF A RF CAVITY}

In order to investigate precisely scenario $n^{\circ} 2$ (transient retuning procedure without stopping the beam), one has first to understand the transient behaviors of the accelerating rf cavities in the linac environment.

\section{A. Modeling the rf cavity + beam}

The spoke and elliptical rf superconducting cavities operate with the $\mathrm{TM}_{010-\pi}$ mode, which produces an accelerating rf voltage on the cavity axis. Using the resistorinductor-capacitor circuit analogy, the behavior of the "cavity + beam" system can be described to first order by the following equation [11]:

$$
\begin{aligned}
\frac{d \tilde{V}_{C}(t)}{d t}= & \frac{\omega(r / Q)}{4}\left[2 \tilde{I}_{G}(t)+\tilde{I}_{B}(t)\right] \\
& -\frac{\omega[1-j \tan \psi(t)]}{2 Q_{L}} \tilde{V}_{C}(t) .
\end{aligned}
$$

$\tilde{V}_{C}$ represents the low frequency component of the accelerating voltage created in the cavity at the operating rf frequency $\omega=2 \pi f$. Its amplitude $V_{C}=\left|\tilde{V}_{C}\right| \approx$ $\left|\int E_{Z}(z) e^{j \omega z / \beta c} d z\right|$ gives the voltage seen by a particle, with velocity $\beta$ and optimal phase, while crossing the cavity (the transit time factor is included). Its phase $\varphi_{c}=$ $\arg \left(\tilde{V}_{C}\right)$ gives the phase of this accelerating voltage, compared to the reference phase of the system which is chosen here to be the phase giving a $0^{\circ}$ synchronous phase. In the case where the cavities are modeled using punctual gaps, this phase is therefore directly equal to the synchronous phase.
$\tilde{I}_{B}$ represents the low frequency component of the beam current crossing the cavity. For short bunches, its amplitude is given by $I_{B}=\left|\tilde{I}_{B}\right| \approx 2 I_{0}$, where $I_{0}$ is the beam mean current. Its phase $\varphi_{B}=\arg \left(\tilde{I}_{B}\right)=\pi$ by definition of the reference phase.

$\tilde{I}_{G}$ represents the low frequency component of the current created by the rf power generator. Its amplitude is given by $I_{G}=\left|\tilde{I}_{G}\right|=2 \sqrt{P_{\text {inc }} /\left[(r / Q) Q_{i}\right]}$, where $P_{\text {inc }}$ is the incident $\mathrm{rf}$ power, $(r / Q)$ the cavity shunt impedance (linac definition), and $Q_{i}$ the incident coupling. Its phase is noted $\varphi_{G}=\arg \left(\tilde{I}_{G}\right)$.

The resonant frequency of the cavity $f_{\text {cav }}$ is always varying because of various perturbations (microphonics, Lorentz detuning, ...), and these perturbations cannot be neglected because of the narrow bandwidth of the loaded cavity. These fluctuations are included in (1) through the detuning angle of the cavity, $\tan [\Psi(t)] \cong 2 Q_{L}\left[f_{\text {cav }}(t)-\right.$ $f] / f$, where $Q_{L}$ is the quality factor of the loaded cavity $\left(1 / Q_{L}=1 / Q_{0}+1 / Q_{i}+1 / Q_{t}\right)$. We choose to describe these frequency fluctuations with a first order model using the following equation (2), where $\Delta f_{\mathrm{CTS}}(t), \Delta f_{L}(t)$, $\sum_{i} \Delta f_{\mathrm{MIC}_{i}}(t) \sin \left(2 \pi f_{\mathrm{MIC}_{i}} t\right)$ are the detuning contributions from cold tuning system management, Lorentz forces, and microphonics, respectively, $k_{L}$ is the Lorentz force detuning coefficient in $\mathrm{Hz} /(\mathrm{MV} / \mathrm{m})^{2}$, and $\tau_{m}$ is the mechanical time constant of the cavity:

$$
\begin{aligned}
f_{\text {cav }}(t)= & f+\Delta f_{L}(t)+\Delta f_{\mathrm{CTS}}(t) \\
& +\sum_{i} \Delta f_{\mathrm{MIC}_{i}}(t) \sin \left(2 \pi f_{\mathrm{MIC}_{i}} t\right)
\end{aligned}
$$

with $\frac{d \Delta f_{L}(t)}{d t}=\frac{1}{\tau_{m}}\left(\frac{10^{-12} k_{L}}{L_{\mathrm{acc}}^{2}} V_{C}^{2}(t)-\Delta f_{L}(t)\right)$.

The solution of the coupled equations (1) and (2) gives the transient evolution of the accelerating voltage $\tilde{V}_{C}(t)$ in the cavity. More details can be found in [12].

\section{B. Modeling the rf regulation loop}

Because of various perturbations (cavity frequency variations, beam transients, ...), the accelerating voltage $\tilde{V}_{C}(t)$ produced in the cavity from (1) and (2) is not stable at all. In order to regulate the accelerating field and phase, a regulation loop is required, called "low-level rf" (LLRF) regulation loop. Its role is to monitor the field produced in the cavity using a capacitive probe, perform an adequate treatment of the signal, compare it to the desired $\left(V_{C}, \varphi_{C}\right)$ set-point, and use the detected error to react on the rf highpower amplifier stage via a proportional/integrative/derivative (PID) controller.

A rough but meaningful modeling of such a loop has been defined. The main elements are (i) a comparator that monitors the probe signal and compare it to the desired setpoint; at this location of the loop, the signals have been digitalized, and the comparison is made using I/Q signals (see $[9,12]$ for more details); (ii) a PID controller (we will 
here only use the gain "P" as a first approach); (iii) a delay and/or a low-pass filter to account for the bandwidth of the whole system; the typical order of magnitude is a few $\mu \mathrm{s}$ delays, and a few $\mathrm{kHz}$ cutoff frequency.

\section{COUPLING RF AND BEAM DYNAMICS}

In order to test the global feedback scheme within scenario $n^{\circ} 2$ (local compensation method with no beam stop), a new tool was needed in order to better understand the transient beam behavior during rf cavity faults, and the right way to recover the correct energy output. The objective here was to develop a tool able to simulate the transient states of a full linac, each accelerating cavity using its own rf control loop. This has been done coupling the cavity model shown in the preceding chapter with a beam transport code including space-charge capability and high level cavity description based on a multigap model or field map elements.

\section{A. Simulation code development}

The cavity model describing the transient rf cavity behavior has first been included into the beam envelope and multiparticle CEA code TRACEWIN [13]. Successful validation tests have been performed to compare the results produced by this new cavity module with previous SIMULINK results.

The TRACEWIN code calculates the transport of the reference particle (envelope) or the beam distribution (multiparticle) through the cavities of the linac, and is by default a "static" tool. It has thus been modified to be able to include the "time" variable, and therefore perform simulations at different times. The architecture of the TRACEWIN code "transient calculation" option is shown in Fig. 3. Several time steps are involved in the process. From the initial condition, at $t=0$, where all the cavities are set to their nominal rf fields and phases, different time-based iterations are performed: (i) every $\delta t_{0}$ (time integration step), a new couple of rf field amplitude and phase is evaluated in each cavity of the linac according to rf cavity model; (ii) every $\delta t_{1}$ (time envelope step, $\delta t_{1} \geq \delta t_{0}$ ), a new beam transport calculation is performed through the linac (envelope transport), using the rf field characteristics (amplitude and phase) obtained at this time in each cavity (which can be modeled either by multigap or field map element); this calculation updates the beam characteristics

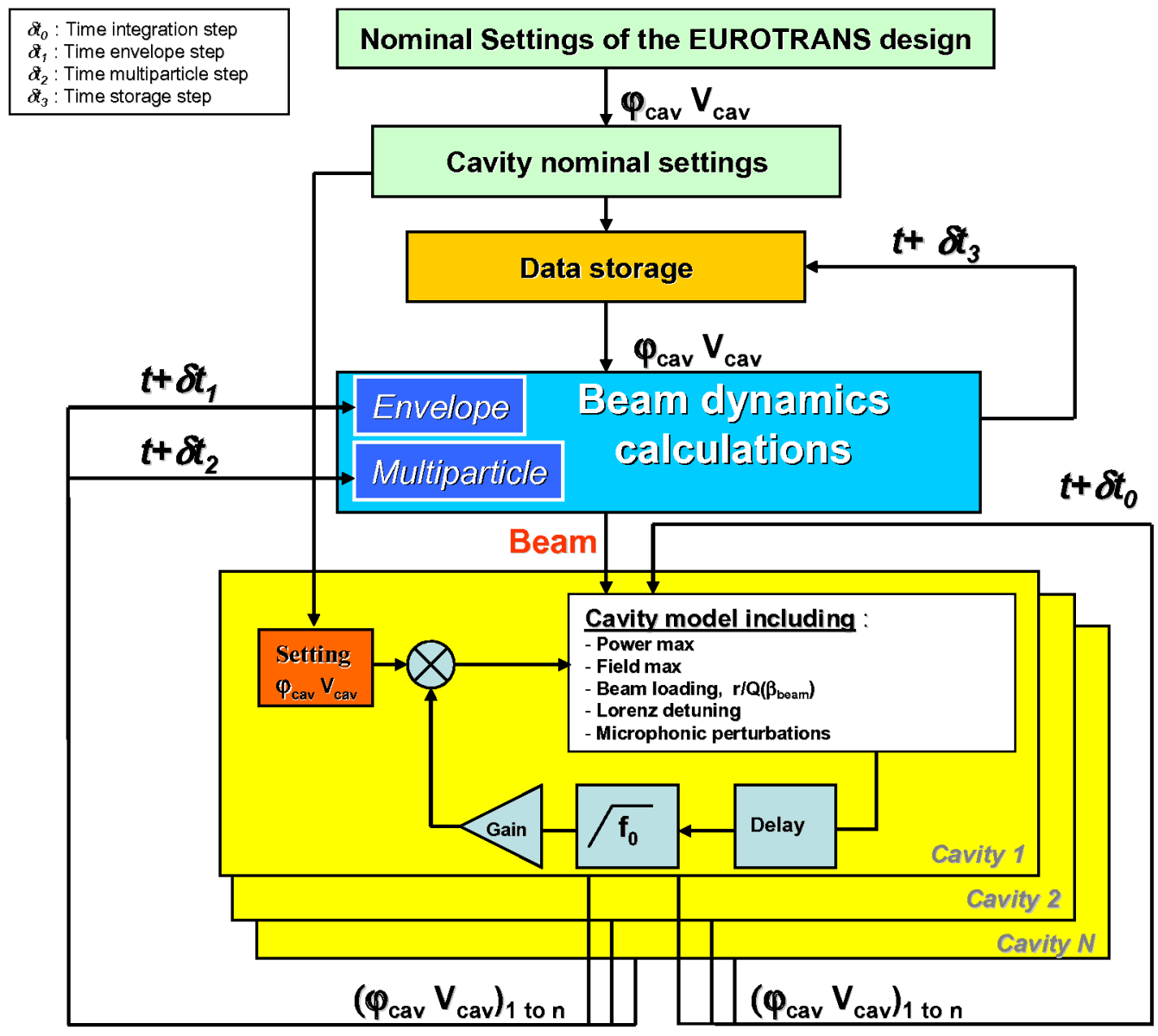

FIG. 3. (Color) Code architecture. 
TABLE II. Cavity and rf loop characteristics used for XT-ADS linac transient calculations.

\begin{tabular}{lccccc}
\hline \hline & Spoke $\beta=0.15$ & Spoke $\beta=0.35$ & Elliptic $\beta=0.47$ & Elliptic $\beta=0.65$ & Elliptic $\beta=0.85$ \\
\hline rf power at saturation $(\mathrm{kW})$ & 15 & 30 & 80 & 150 & 150 \\
Accelerating field margin $(\%)$ & 50 & 50 & 50 & 50 & 50 \\
$r / Q$ at optimal beta $(\Omega)$ & 101 & 220 & 153 & 316 & 598 \\
Form factor $(\Omega)$ & 72 & 101 & 101 & 194 & 300 \\
Lorenz factor $\left(\mathrm{Hz} /(\mathrm{MV} / \mathrm{m})^{2}\right)$ & -8 & -8 & -8 & -8 & -2 \\
Feedback delay $(\mathrm{s})$ & $2 \times 10^{-6}$ & $2 \times 10^{-6}$ & $2 \times 10^{-6}$ & $2 \times 10^{-6}$ & $2 \times 10^{-6}$ \\
Feedback gain & 20 & 20 & 100 & 100 & 100 \\
Low filter frequency $(\mathrm{kHz})$ & 10 & 10 & 10 & 10 & 10 \\
Cryogenic operating $T(\mathrm{~K})$ & 4.2 & 4.2 & 2 & 2 & 2 \\
\hline \hline
\end{tabular}

at each linac location; (iii) every $\delta t_{2}$ (time multiparticle step, $\delta t_{2} \geq \delta t_{1}$ ), a multiparticle transport simulation is performed; this calculation updates the beam characteristics at each linac location; (iv) every $\delta t_{3}$ (time storage step, $\delta t_{3} \geq \delta t_{2}$ ), all the linac and beam characteristics at each location are stored.

The computation time strongly depends on the choice of these different time steps, and especially on $\delta t_{1}$ and $\delta t_{2}$; $\delta t_{3}$ is fixed by the available memory. Typically, to simulate accurately a $10 \mathrm{~ms}$ linac behavior, the time steps are respectively chosen to $\delta t_{0}=1 \mathrm{~ns}, \delta t_{1}=1 \mu \mathrm{s}, \delta t_{2}=$ $10 \mu \mathrm{s}$, and $\delta t_{3}=10 \mu \mathrm{s}$. With 10000 macroparticles, about $1 \mathrm{~Gb}$ memory is needed to save the results, and the simulation spends about 22 hours on a usual PC.

Finally, let us note that, for each kind of cavity of the linac, a file has to be created in order to indicate to the transport code its main characteristics. These files have to contain also the feedback loop parameters, which can be different according to the cavity type. All these data are summarized in Table II in the case of our XT-ADS reference linac. Some extra parameters as microphonic frequencies and amplitudes can be added if needed.

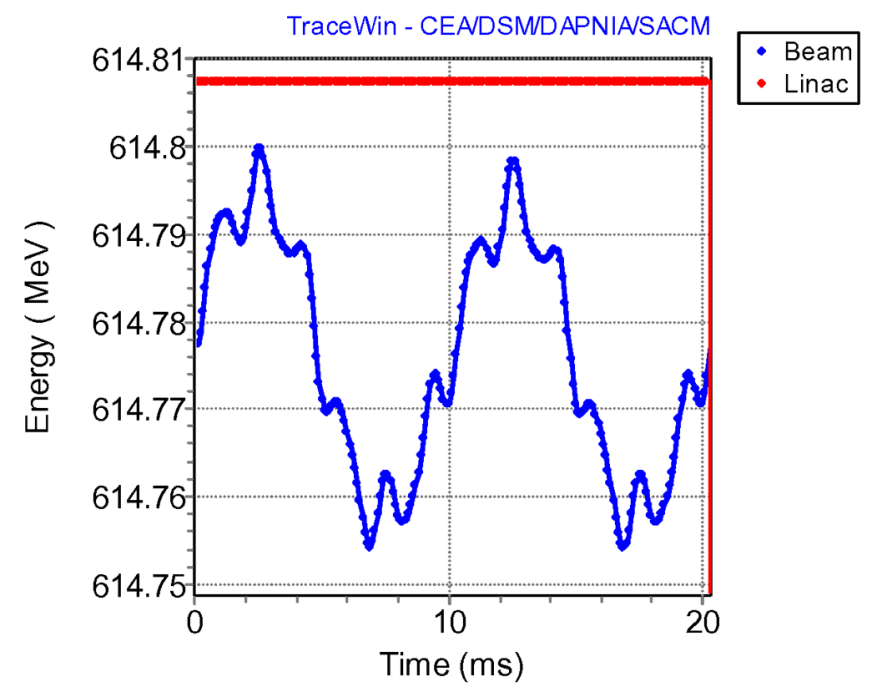

FIG. 4. (Color) Output energy of the last linac cavity with microphonics perturbations.

\section{B. Code capabilities}

Obviously, this new simulation code, which is a quite unique tool, potentially allows performing a lot of different studies on the linac response to various transient phenomenons: influence of microphonics, LLRF feedback quality in presence of beam hole, etc., ....

Figure 4 shows, for example, the output energy of the first and last cavity of the EUROTRANS linac in the case where 3 microphonic fluctuations $(100 \mathrm{~Hz}, 600 \mathrm{~Hz}$, and $1000 \mathrm{~Hz}$ ) of different amplitudes are present in the linac cavities (rf control loop on). Only the microphonic phases are randomly set. The red curve called "Linac" represents the reference static case, and the blue one called "Beam" represents the "transient" case (here: with microphonics) ${ }^{1}$.

Within the present study, this new tool will be used to analyze, on the beam dynamics point of view, the feasibility of a cavity-fault recovery procedure without stopping the beam (scenario $n^{\circ} 2$ ). To this respect, the global feedback scheme has also been implemented in the code. It is based on the same concept that was used in our preceding static fault-tolerance analysis [5]: a few lattices downstream of the faulty cavity, the beam energy and phase are monitored, and the cavities surrounding the failed one are retuned (fields and phases corrections are optimized via iterative calculations) to recover the nominal beam energy and phase at the monitoring location. The only difference is that, in this new transient study, the field and phase correction is not performed one single time per fault condition, like in the static case, but for each time step $\delta t_{2}$.

\section{RF CAVITY FAULT: SIMULATION RESULTS}

The goal here is not to study all the cases of cavity fault, but just to demonstrate a concept. For this reason, the analysis of the fast cavity-fault recovery procedure without stopping the beam (scenario $n^{\circ} 2$ ) is performed for the following case: failure of spoke cavity $\# 92(\beta=0.35)$

\footnotetext{
${ }^{1}$ In the full following document, all the charts will always show these two curves: red $=$ static case; blue $=$ transient case (i.e. with cavity failure).
} 


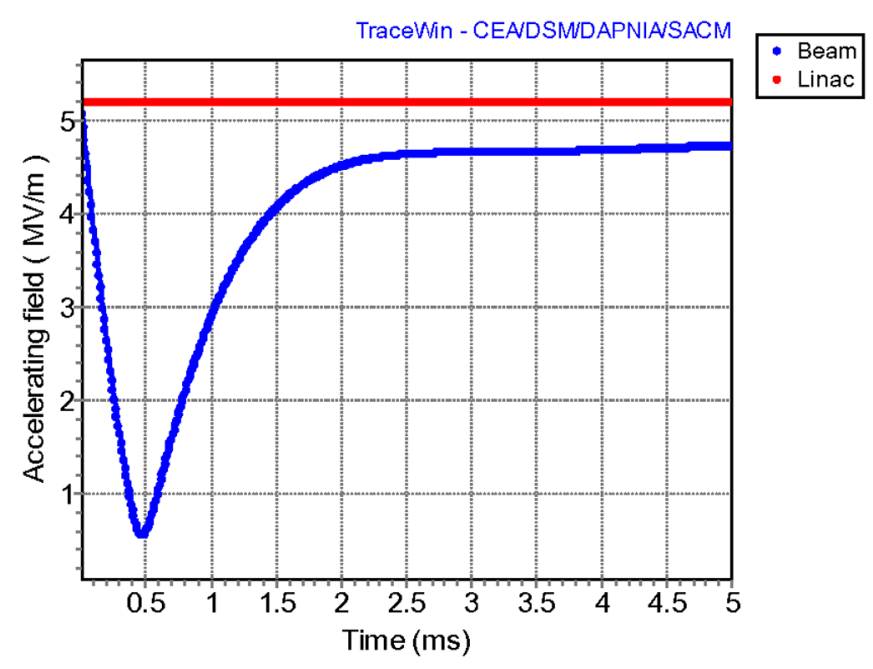

FIG. 5. (Color) Field in the failed cavity.

located near the end of the second linac section. The considered beam current is $10 \mathrm{~mA}$, corresponding to a nominal $\sim 15 \mathrm{~kW}$ to give to the beam. We will only concentrate on this specific case.

\section{A. RF cavity failure without compensation}

The goal here is to study the linac and beam behavior when a cavity breaks down. All cavities are simulated with their feedback loop closed, and we consider the following timing: (i) $t=0$, all cavities of the linac are set at the nominal field and power. The energy output is stable according to time. (ii) $t=0+\varepsilon, \varepsilon$ being negligible, a rf cavity/amplifier breaks down (rf power off); the associated feedback loop is then opened.

The analysis of the beam behavior in this case where nothing is done to recover the situation will help us to determine the time we have to react and perform the first

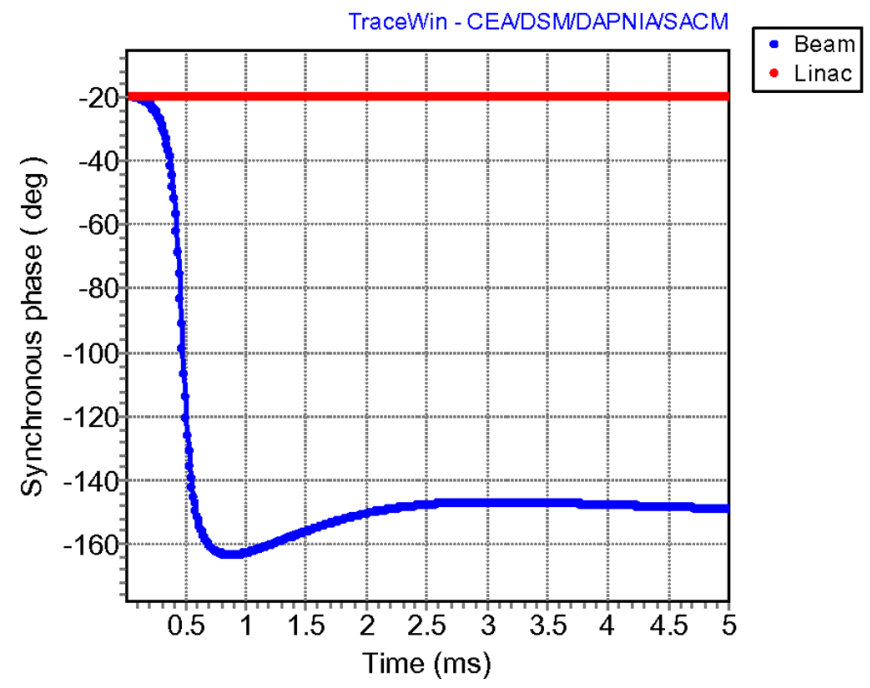

FIG. 6. (Color) Synchronous phase in the failed cavity.

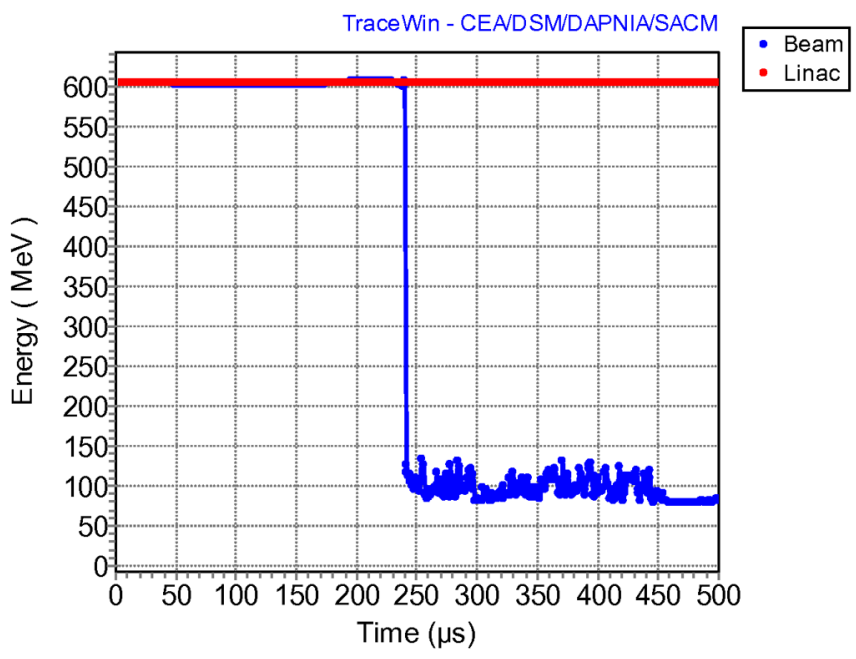

FIG. 7. (Color) Output beam energy at the linac end.

compensations in order to conserve the beam with good parameters (emittances, energy, phase, ....).

The results are shown in the following figures. Figures 5 and 6 show the transient evolution of the beam synchronous phase and of the accelerating field in the failed cavity. After about $500 \mu \mathrm{s}$, the field is coming close to 0 , and after $2.5 \mathrm{~ms}$ the cavity becomes a decelerating cavity (see the fast phase shift), producing a decelerating field roughly equal to the nominal accelerating field. This is due to the beam loading effect, and it happens because we do not have the time in scenario $n^{\circ} 2$ to detune the failed cavity to put if off frequency. This is a major difference with scenario $n^{\circ} 1$,
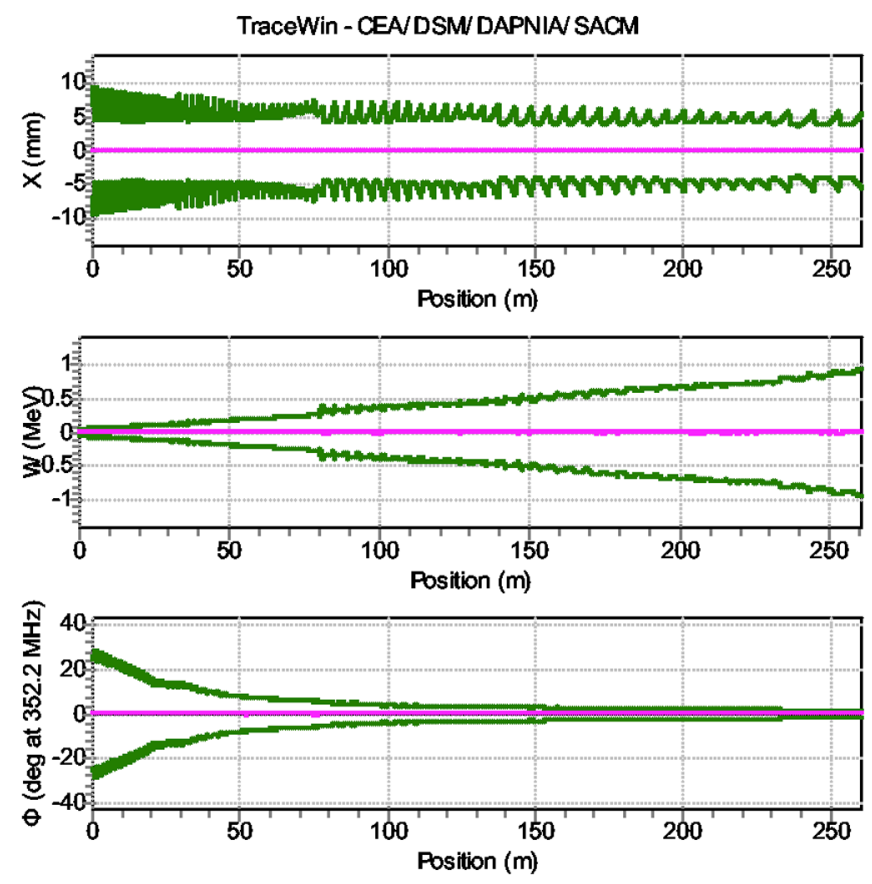

FIG. 8. Envelopes at $0 \mu \mathrm{s}$, reference linac. 

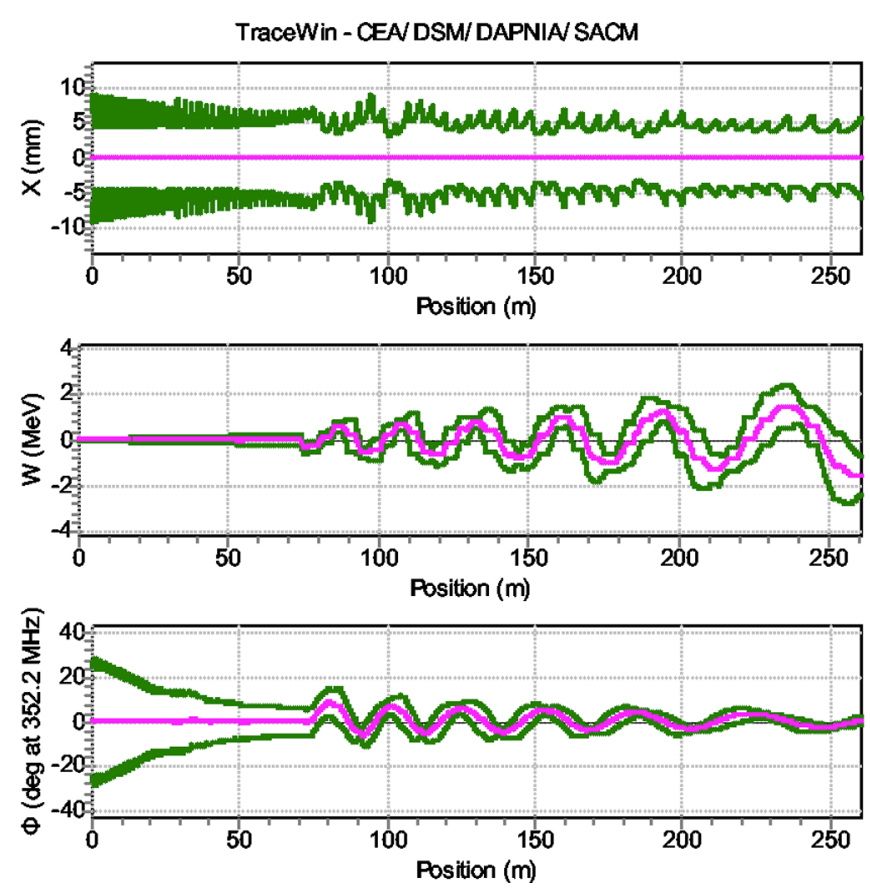

FIG. 9. Envelopes at $100 \mu \mathrm{s}$.

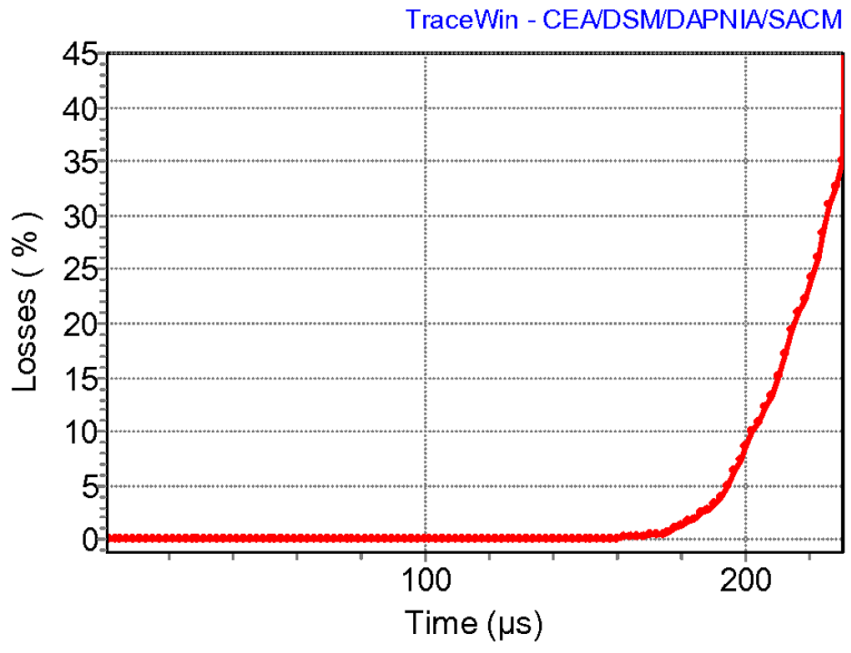

FIG. 10. (Color) Beam losses according to time.

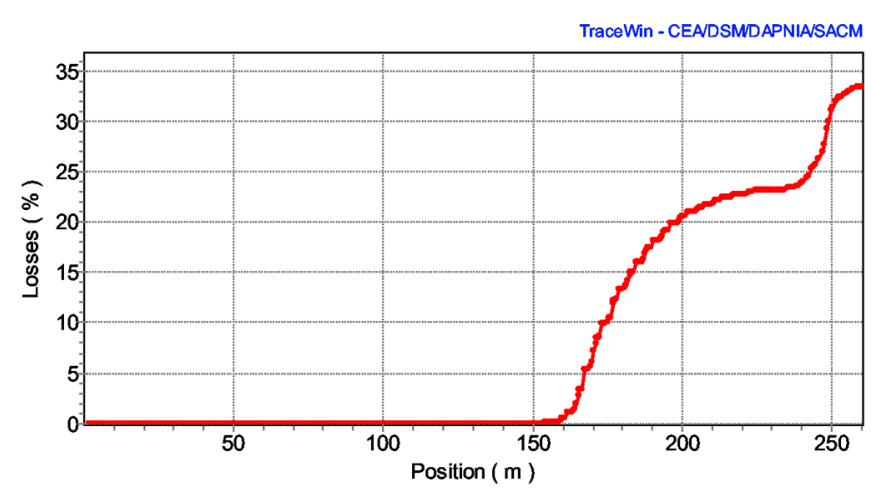

FIG. 11. (Color) Losses along the linac at $220 \mu$ s. in which we can detune the cavity during the $1 \mathrm{~s}$ beam hole and avoid this decelerating effect at the beam reinjection.

The consequence to the output beam energy is shown on Fig. 7, which shows the beam going out of the linac acceptance after about $240 \mu \mathrm{s}$. The final energy output is then stabilized around $90 \mathrm{MeV}$, which is already the beam energy at the failed cavity.

The new tool also allows studying the beam transverse behavior with respect to time. Figures 8 and 9 illustrate, for example, the beam envelope at different times. Multiparticle simulations show that the first beam losses are observed after $160 \mu$ s (cf. Fig. 10), and Fig. 11, plotted at $220 \mu \mathrm{s}$, show beam losses of about $33 \%$ distributed all along the linac end (failed cavity position is about 75 meters), Fig. 12 show the output beam distributions at $200 \mu \mathrm{s}$. We have to note that TRACEWIN considers a particle as lost only when it reaches the beam tube (no cutoff in energy or phase is used here).

These results mean that, if we want to retune some cavities so as to keep the entire beam during the whole procedure, we will have to start it, in this specific case, $160 \mu \mathrm{s}$ at maximum after the cavity failure.

\section{B. RF cavity failure with compensation}

The procedure to conserve the beam is based on the following timing: (i) $t=0$ : the rf cavity/amplifier breaks down (rf power off); the associated feedback loop is then opened and the cavity field starts to decrease. (ii) $t=T_{1}$ : first couples of set-points $\left(V_{C}, \varphi_{C}\right)$ are sent to the adjacent cavities, computed from the situation at $T_{1} . T_{1}$ represents the time needed to detect the failure, and has to be short enough to conserve a correct beam transport. (iii) $t=T_{1}+$ $T_{2}$ : second couples of set-points $\left(V_{C}, \varphi_{C}\right)$ are sent to the adjacent cavities, computed from the situation at $T_{1}+T_{2}$. (iv) Every $T_{2}$, new couples of set-points $\left(V_{C}, \varphi_{C}\right)$ are sent to the adjacent cavities, until the beam behavior becomes stable in phase and energy. $T_{2}$ has also to be short enough to conserve a correct beam transport.

In the simulation, at each $T_{2}$ step, couples of new setpoints $\left(V_{C}, \varphi_{C}\right)$ are estimated by an optimization obtained by iteration from the beam phase and energy monitoring. As already mentioned, for the real accelerator operation, these sets of retuning values will have to be precalculated by simulations, and stored in a table in order to be able to be applied very quickly when needed. This means that, once the linac design is frozen, each case of cavity failure will have to be studied beforehand.

The results presented next are still given for the spoke cavity \#92 failure. Several configurations have been looked at, with different numbers of correcting cavities, available power margins, accelerating field margins, or different $T_{1}$ and $T_{2}$. Only one case is presented in the following, corresponding to the following hypotheses: (i) $30 \mathrm{~kW} \mathrm{rf}$ amplifiers saturation level (see Table II); (ii) 50\% accelerating field margin (see Table II); (iii) 6 spoke cavities 


\section{NGOOD : 85164/85164 I=8.5 ms. Plotwin - CEA/DSM/DAPN/A/SACM}
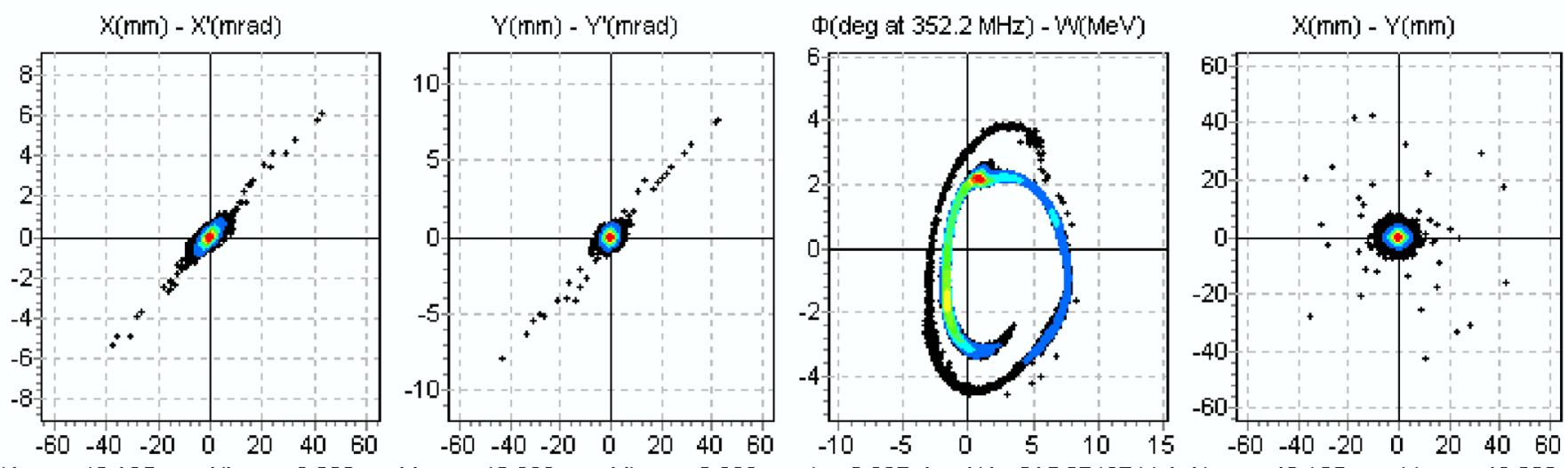

Xmax $=43.135 \mathrm{~mm} X^{\prime} \max =6.083 \mathrm{mr}$ Ymax $=43.086 \mathrm{~mm} \quad Y^{\prime} \operatorname{rnax}=8.030 \mathrm{~m}$

$\Phi_{0}=0.337$ deg 'Wo=615.37427 Me' $X_{\max }=43.135 \mathrm{mmn} \mathrm{Y}_{\max }=43.086$

FIG. 12. (Color) Beam distributions output at $200 \mu \mathrm{s}, 10 \%$ of particle left linac acceptance.

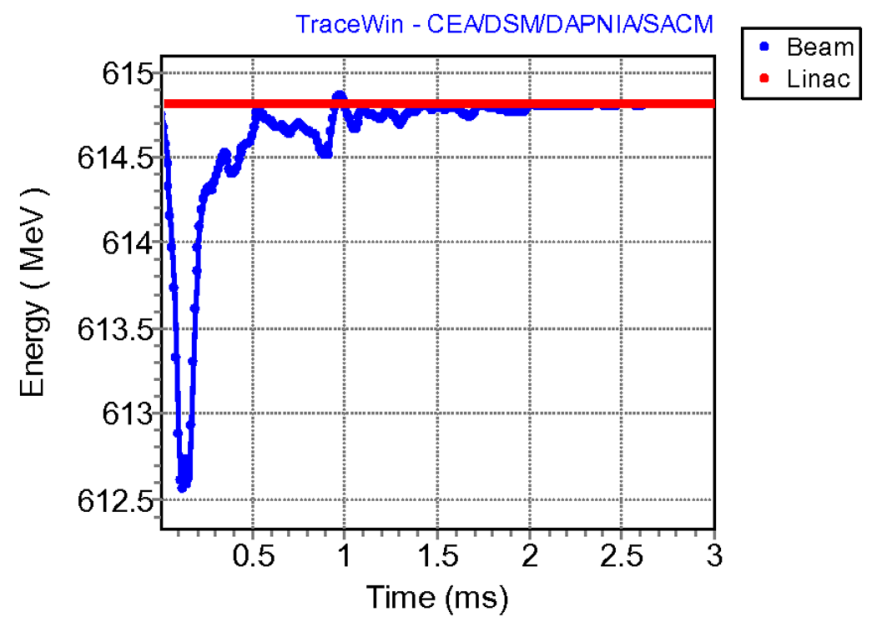

FIG. 13. (Color) Output beam energy.

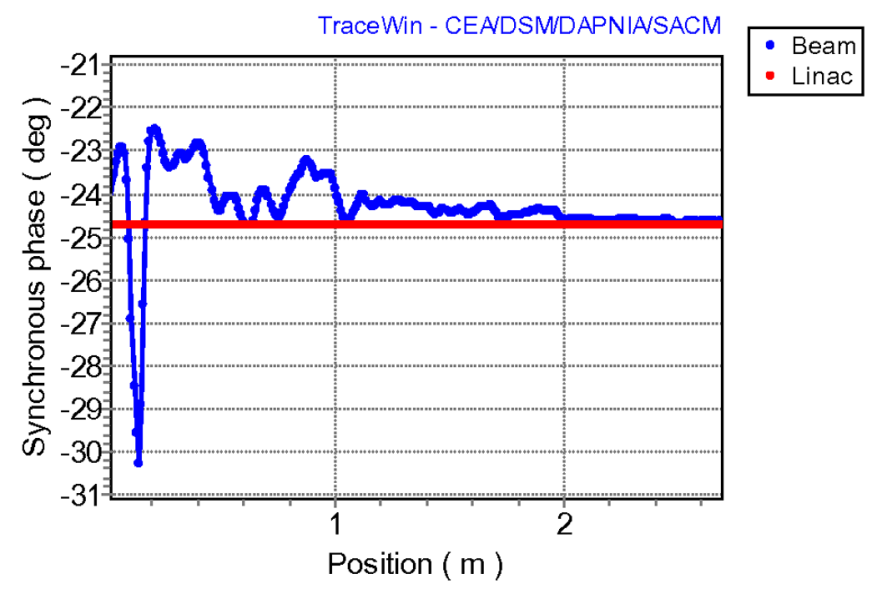

FIG. 14. (Color) Beam synchronous phase at last cavity.
( 3 before +3 after) used to compensate the cavity failure. If the accelerating field margin is reduced to $40 \%$, then 8 neighboring cavities are needed.

As already discussed, the failed cavity is not detuned in this scenario $n^{\circ} 2$, and thus produces a decelerating field due to the beam loading effect. This is why more cavities and/or margins are needed here than in scenario $n^{\circ} 1$, in which less than $30 \%$ field margins for the same retuning with $3+3$ cavities, and $\sim 25 \mathrm{~kW}$ amplifiers could be used in this section [5].

Given these parameters, it can be shown that a transient fault-recovery procedure without stopping the beam is manageable without any beam loss. (i) 40 corrections have been calculated and set to neighboring cavities during the whole retuning procedure. (ii) The final beam energy is recovered and stable after $3 \mathrm{~ms}$ (Fig. 13). The maximum energy error is about $2.2 \mathrm{MeV}$ at $140 \mu \mathrm{s}$, which gives a maximum power fluctuation at the target of $0.5 \%$. At $140 \mu \mathrm{s}$, we also record the maximum beam synchronous

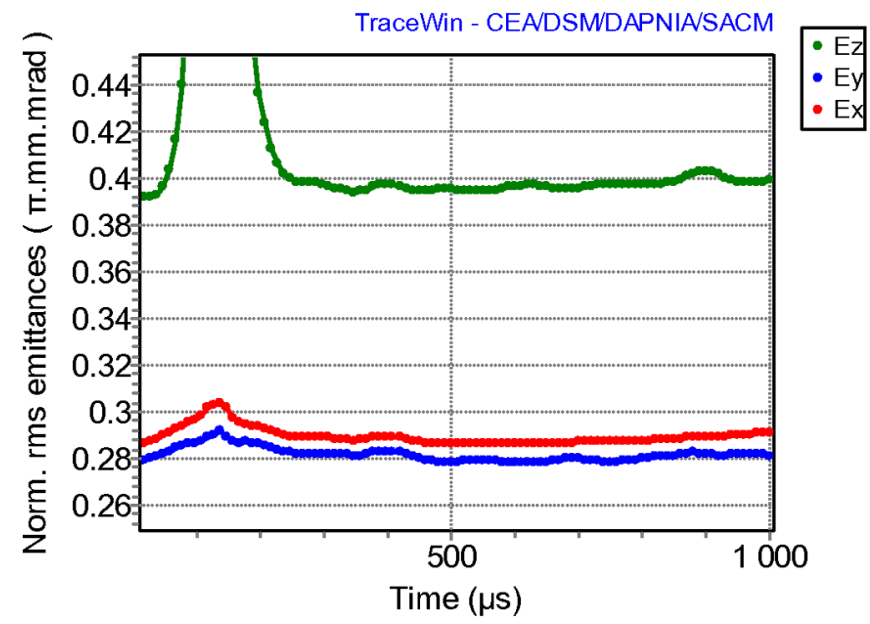

FIG. 15. (Color) Emittance evolution during the first ms. 
Ele: 779 [260.481 m] NG00D : $100000 / 100000$ TraceWin - CEA/DSW/DAFN/A/SACW
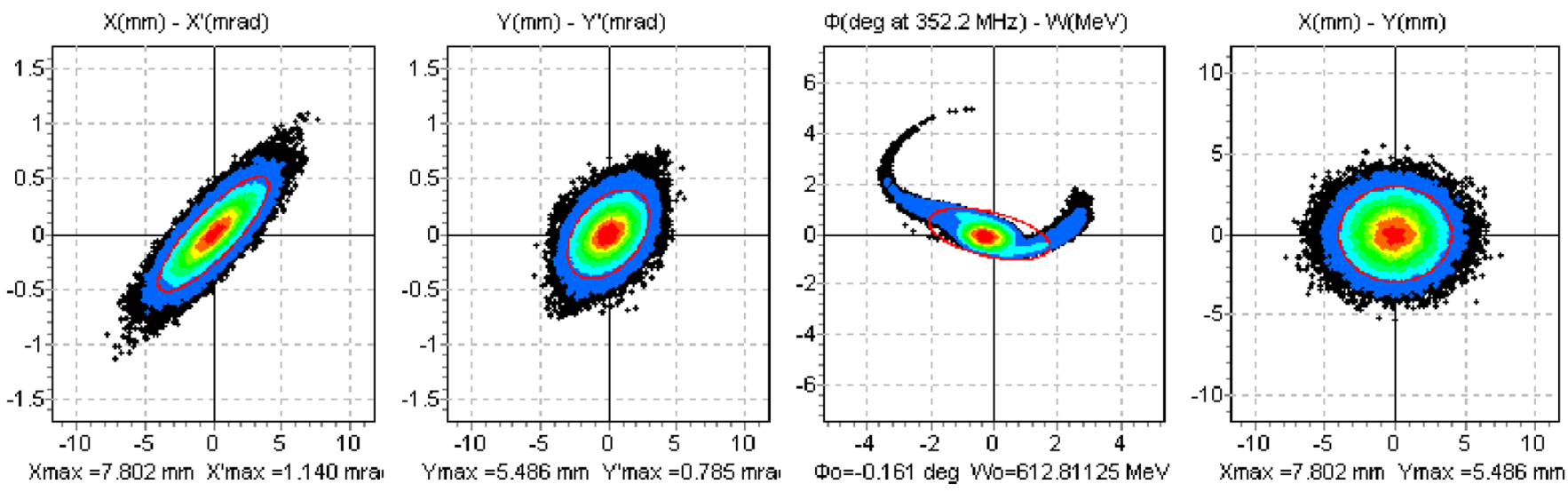

FIG. 16. (Color) Output beam phase spaces at $140 \mu \mathrm{s}$.

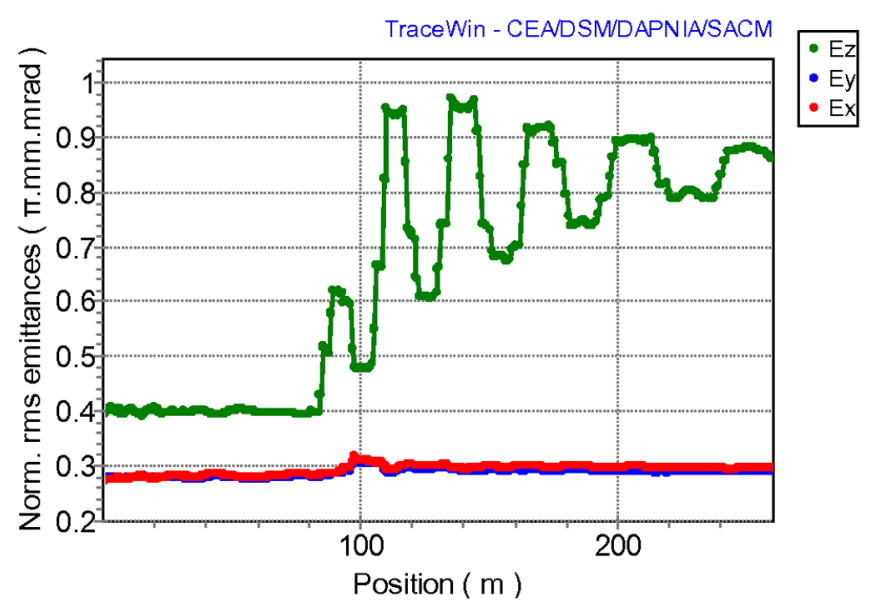

phase error $\left(5^{\circ}\right)$ in the last cavity of the linac (Fig. 14). (iii) Less than $10 \%$ of transverse emittance growth is observed (Fig. 15), with no beam loss observed during the $3 \mathrm{~ms}$ of simulation with 10000 macroparticles. (iv) The worse beam transport occurs at $140 \mu \mathrm{s}$. Figure 16 shows the output space phase beam at $140 \mu \mathrm{s}$ and Fig. 17 shows the emittance behavior along the linac at this time. We clearly see the longitudinal emittance increasing, but the beam is still sufficiently well transported allowing one to get at the output only $10 \%$ transverse emittance growth.

Finally, the Fig. 18 represents an example of the field evolution in the first cavity of the six cavities used to compensate the failed cavity.

FIG. 17. (Color) Emittances along the linac at $140 \mu \mathrm{s}$.

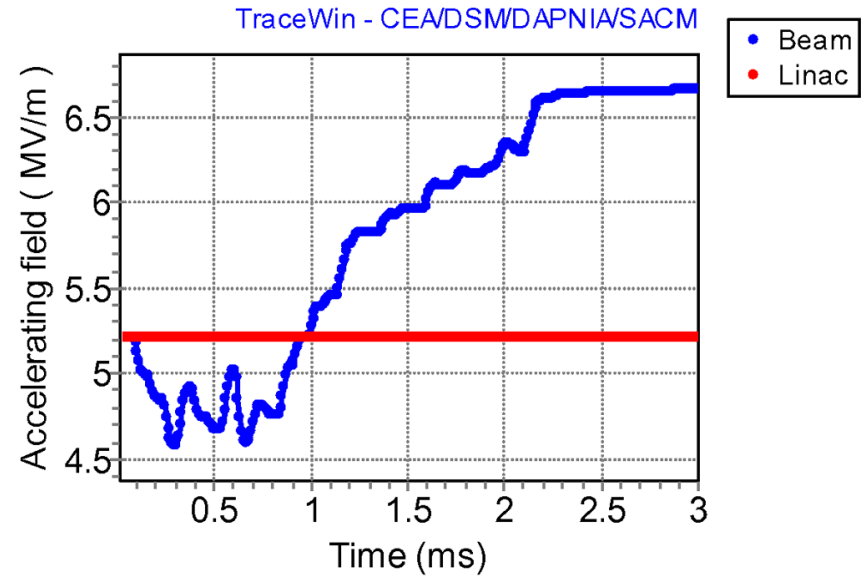

FIG. 18. (Color) Field in cavity \#1.
TraceWin - CEAJDSMDAPNIASACM

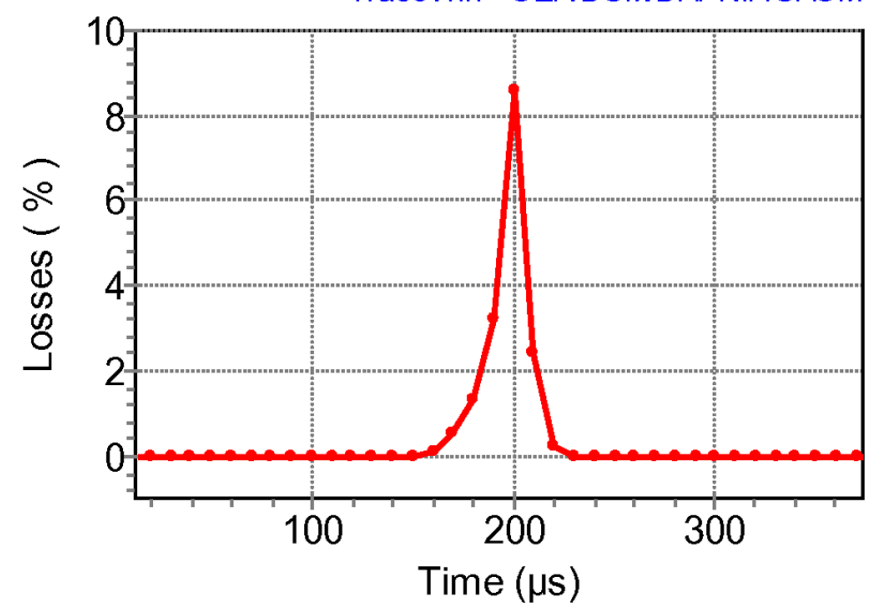

FIG. 19. (Color) Beam losses in function of time for $T_{1}=T_{2}=$ $200 \mu \mathrm{s}$. 
TABLE III. Requirements and features of the two fault-recovery scenarios.

\begin{tabular}{ll}
\hline \hline \multicolumn{1}{c}{ Scenario $n^{\circ} 1$ (stopping the beam) } & \multicolumn{1}{c}{ Scenario $n^{\circ} 2$ (on-line) } \\
\hline Implies a $\leq 1$ sec beamstop & No beamstop \\
"Relatively fast" fault time detection & "Extremely fast" fault time detection \\
Lower field/power or number of & Higher field/power or number of \\
cavities are required & cavities are required \\
Small predefined set-points database & Huge predefined set-points database \\
Fast cold tuner management required & Classical cold tuner management \\
\hline \hline
\end{tabular}

\section{Time limits}

In this specific example, the time limit to start correction is $150 \mu \mathrm{s}$. If the compensation procedure starts later, the beam begins to get out of the linac acceptance and beam losses appear. The correction step has also to be smaller than $150 \mu \mathrm{s}$.

This is illustrated in Fig. 19, that shows the beam losses as a function of time when the procedure starts at $t=$ $200 \mu \mathrm{s}$ : some transient beam losses are clearly observed during the recovery procedure.

\section{CONCLUSION}

The present analysis demonstrates from the beam dynamics point of view that a fast retuning procedure can be envisaged without stopping the beam. This is what we called scenario $n^{\circ} 2$.

Nevertheless, this scenario $n^{\circ} 2$ implies stringent specifications, especially (i) the fault detection time has to be extremely short (order of magnitude: $100 \mu \mathrm{s}$ ); this means that the rf control loop has to include some dedicated diagnostics and/or algorithms capable to detect (or anticipate) any rf malfunctioning and inform as quickly as possible the global feedback system; (ii) to apply the fault-recovery scenario $n^{\circ} 2$, higher accelerating field and rf power are required, compared to the scenario $n^{\circ} 1$ where the beam is stopped and the cavity detuned; this point could have some impact on the cost point of view.

Table III summarizes the main requirements and features for the two envisaged fault-recovery scenarios. Both scenarios are feasible on the beam dynamics point of view. The choice will first rely on the results of research and development activities to be performed on (i) fast rf fault anticipation/detection, (ii) fast cold tuning system management, and (iii) fast LLRF communication and data exchange
Once these points are clarified, integrated reliability analysis and cost statements will have to be performed, so as to optimize the fault-recovery scenario process in terms of number of cavities involved, of accelerating field margins, and of rf power budget.

\section{ACKNOWLEDGMENTS}

This work is performed within the 6th Framework Program EC project "EUROTRANS" (EC Contract No. FI6W 516520, EUROTRANS).

[1] H. Safa, A.C. Mueller, and B. Carluec, PDS-XADS internal report, Deliverable 9, 2002.

[2] J.-L. Biarrotte, A. C. Mueller, and B. Carluec, PDS-XADS internal report, Deliverable 63, 2004.

[3] J.-L. Biarrotte et al., Nucl. Instrum. Methods Phys. Res., Sect. A 562, 656 (2006).

[4] J.-L. Biarrotte and A.C. Mueller, in Proceedings of the ICENES Conference, Istambul, 2007.

[5] J.-L. Biarrotte, M. Novati, P. Pierini, and D. Uriot, in Proceedings of the HPPA4 Workshop, Daejon, 2004.

[6] P. Pierini, in Proceedings of the HPPA5 Workshop, Mol, 2007.

[7] J. Galambos et al., in Proceedings of the HPPA5 Workshop, Mol, 2007.

[8] J. Galambos (personal communication).

[9] M. Luong et al., EUROTRANS internal report, Deliverable 1.40, 2007.

[10] C. Joly et al., LLRF07 Workshop, Knoxville, 2007.

[11] T. Schilcher, TESLA Report No. 98-20, Hamburg, 1998.

[12] J.-L . Biarrotte, IPNO Report No. 07-06, Orsay, 2007.

[13] R. Duperrier, N. Pichoff, and D. Uriot, in Proceedings of the ICCS Conference, Amsterdam, 2002. 